\title{
SHORTER NOTICES
}

Algebra. By Oskar Perron. Berlin and Leipzig, Walter de Gruyter, 1927. Two volumes. Volume I. Die Grundlagen. 4 figures; viii $+307 \mathrm{pp}$. Volume II. Theorie der algebraischen Gleichungen. 5 figures; viii +243 pp. Göschens Lehrbucherei, I. Gruppe: Reine Mathematik, Band 8, 9.

In view of the large number of texts on higher algebra already on the market, the average mathematician is not ant to hail a new book on this subject with joy or even interest. As one's eye runs hastily down the table of contents and sees a sequence of titles that calls to mind a composite picture of Weber, Serret, Burnside and Panton, et al., one can certainly be excused if one does not at first wax enthusiastic. In fact, the chapterheadings in Volume I are, in order: fundamental notions; polynomial theorem and Taylor's theorem; determinants (including the elements of matrices and of bilinear, quadratic and Hermitian forms); symmetric functions; divisibility of polynomials; existence of roots; and in Volume II the chapter-headings are: numerical solution of equations; reciprocal equations and equations of degree $\geqq 4$; substitution groups; Galois' theory of equations; equations of degree 5. Moreover, the captions of the subdivisions of the eleven chapters are, generally, the classic ones. Thus, first impressions lead one to expect merely one more book to add to the library catalogue and to which a beginning graduate student will look for help when Weber, Serret et al. happen to be in use. But, fortunately, first impressions are unjust in this case. For there are two places in the book where the treatment is sufficiently different from the classic treatment to interest a reader familiar with the usual treatise on the subject.

Probably the outstanding characteristic of the book is the prominence which is given to the notion of field (domain of rationality). This is certainly good pedagogy, for this is one of the most important concepts in mathematics. To be sure, the most central notion of all algebra is that of a linear algebra (hypercomplex numbers) including as special cases fields, groups, matrices and integral equations. But it would be too much to expect the author of a comparatively elementary text to display his various topics as special cases or applications of linear algebras; and we are glad to see that he has clustered the more strictly algebraic parts of his book around the notion of field. In particular, he has presented the subject of the divisibility of polynomials in one or more variables in such a way that there are not those annoying exceptions in the statement of the theorems about the highest common factor of two polynomials in two or more variables, as in Bôcher's text. Also, the emphasis on fields in the first volume admirably prepares the way for the discussion of Galois' theory of equations in the second volume. Although the notion of field is always used in this subject, of necessity, yet the author's presentation is such as to give to the student a number of important theorems about algebraic fields which he does not usually see formulated outside a course in algebraic numbers 
and should give him a grasp of the elements of this subject such as he does not usually have after taking the usual course in Galois' theory of equations. This aspect of the book is highly to be commended.

On the other hand, when the author has departed to this extent from the precedent set by Weber, Serret et al., it is all the more disappointing to find that he includes the classic material on the numerical solution of equations-Sturm's theorem, the theorem of Fourier and Budan, Descartes' rule of signs, upper limits of the roots, Newton's method, "regula falsi," and the methods of approximation due to Bernoulli and Gräffe, although he is wise enough to omit Horner's method. Mathematics of this kind certainly does not belong in the same book with a neat and clear exposition of the divisibility of polynomials in several variables and Galois' theory of equations except in so far as it may be used to illustrate a bit of theory. In fact, such mathematics is not algebra at all, but what one might call "algebraic statistics"- that is, it applies to concrete problems in algebra the methods of statistics rather than the methods of pure algebra.

O. C. HazLETt

Sur la Théorie des Equations aux Dérivếes Partielles du Premier Ordre d'une Seule Fonction Inconnue. By N. N. Saltykow. Paris, Gauthier-Villars, 1925. $172 \mathrm{pp}$.

This book contains the lectures delivered by the author in the four Belgian Universities during 1923-1924. Much of the material represents results of the author's own researches which have not previously been published in detail or which are now presented in simplified form.

The treatment centers about the well known theorem concerning the equivalence of the two problems: (1) to find a complete integral of a given partial differential equation of the first order in one unknown function; (2) to find the general integral of the equations of its characteristics. The author develops a theory which exhibits the relations between these problems and which gives methods of finding a solution of either one when any incomplete set of integrals of the equations of the characteristics is known. He extends his results, moreover, to simultaneous systems by proofs like those for the single equation. In fact, the similarity is such that one wonders why he does not treat the more general case at the outset.

Since the characteristics can be defined by a linear homogeneous system of partial differential equations, there is an introductory chapter on the theory of such systems. In particular, a new form of the equations of the characteristics, fundamental for the subsequent developments, results from this preliminary discussion.

The contributions are both interesting and important. The details of the exposition could perhaps be improved upon. A clear-cut statement of hypothesis and conclusion would help. For example, at the beginning of Chapter II the author announces he is about to prove Jacobi's theorem, but leaves the reader to find out which one. The misprints are numerous but of little consequence.

J. M. Thomas, National Research Fellow 